Pacific

Journal of

Mathematics

\title{
STEINITZ CLASS OF MORDELL-WEIL GROUPS OF ELLIPTIC CURVES WITH COMPLEX MULTIPLICATION
}

Tong LiU and Xianke Zhang 


\title{
STEINITZ CLASS OF MORDELL-WEIL GROUPS OF ELLIPTIC CURVES WITH COMPLEX MULTIPLICATION
}

\author{
TONG LiU AND Xianke Zhang
}

\begin{abstract}
Let $E$ be an elliptic curve having Complex Multiplication by the ring $\mathcal{O}_{K}$ of integers of $K=\mathrm{Q}(\sqrt{-D})$, let $H=K(j(E))$ be the Hilbert class field of $K$. Then the Mordell-Weil group $E(H)$ is an $\mathcal{O}_{K}$-module. Its Steinitz class $\mathrm{St}(E)$ is studied here. In particular, when $D$ is a prime number, $\operatorname{St}(E)$ is determined: If $D \equiv 3(\bmod 4)$ then $\mathrm{St}(E)=1$; if $D \equiv 1$ $(\bmod 4)$ then $\operatorname{St}(E)=[\mathcal{P}]^{t}$, where $\mathcal{P}$ is any prime-ideal factor of 2 in $K,[\mathcal{P}]$ the ideal class of $K$ represented by $\mathcal{P}, t$ is a fixed integer. In addition, general structure for modules over Dedekind domain is also discussed. These results develop the results by $D$. Dummit and W. Miller for $D=10$ and specific elliptic curves to more general $D$ and general elliptic curves.
\end{abstract}

\section{Introduction.}

Let $K=\mathbf{Q}(\sqrt{-D})$ be an imaginary quadratic number field, $\mathcal{O}_{K}$ the ring of all integers of $K$. Let $E$ be an elliptic curve having Complex Multiplication by the ring $\mathcal{O}_{K}$. Then $E$ is defined over the field $F=\mathbf{Q}(j(E))$, where $j(E)$ denotes the $j$-invariant of $E$. So $H=K(j(E))$ is the Hilbert class field of $K$, [4], and the Mordell-Weil group $E(H)$ (i.e., all the $H$-rational points of $E$ ) is naturally a module over the Dedekind domain $\mathcal{O}_{K}$ (operation is the complex multiplication). By the structural theorem for finitely generated modules over Dedekind domain we have

$$
E(H) \cong E(H)_{\text {tor }} \oplus \mathcal{O}_{K} \oplus \cdots \oplus \mathcal{O}_{K} \oplus \mathcal{A}=E(H)_{\text {tor }} \oplus \mathcal{O}_{K}^{s-1} \oplus \mathcal{A},
$$

where $\mathcal{A}$ is an ideal of $\mathcal{O}_{K}$ which is uniquely determined by $E(H)$ up to a multiplication by a number from $K$. Thus $E(H)$ determines uniquely an ideal class $[\mathcal{A}]$ of $K$ represented by $\mathcal{A} ;[\mathcal{A}]$ is said to be the Steinitz class of $E$ and denoted by $\operatorname{St}(E)$. (Similarly, any module $M$ over a Dedekind domain $R$ defines an ideal class of $R$, which is said to be the Steinitz class of $M$ and denoted by $\operatorname{St}(M)$.) So the structure of the Mordell-Weil group $E(H)$, as a module over the Dedekind domain $\mathcal{O}_{K}$, is uniquely determined by its Steinitz class, rank $s$, and its torsion part. Therefore, it is important to determine the Steinitz class. D. Dummit and W. Miller, [1] in 1996 
determined the Steinitz class of some specific elliptic curves when $D=10$ and found some of their properties.

Since the Steinitz class $\operatorname{St}(E)$ is essentially concerned only with the free part of $E(H)$, we denote

$$
E(\cdot)_{f}=E(\cdot) / E(\cdot)_{\text {tor }},
$$

that is, the quotient group of the Mordell group $E(\cdot)$ modulo its torsion part. Note that $E(\cdot)_{f}$ is isomorphic to the free part of $E(\cdot)$. This notation will be used also for any subgroup I of $E(\cdot)$ to define $I_{f}$. Also we can assume the Weierstrass equation of the elliptic curve $E$ to be ([5])

$$
E: y^{2}=f(x)=x^{3}+a_{2} x^{2}+a_{4} x+a_{6}
$$

with $a_{2}, a_{4}, a_{6} \in F$.

We will first analyze the interior structure of $E(H)$, give a general theorem for the structure of modules over Dedekind domain, and then determine Steinitz classes $\operatorname{St}(E)$ for some types of elliptic curves. In particular, when $D=p$ is a prime number and $p \equiv 3(\bmod 4)$, we will prove that $\operatorname{St}(E)$ is the principal class of $K$; And when $D=p$ is a prime number and $p \equiv 1$ $(\bmod 4)$, we will show that

$$
\operatorname{St}(E)=[\mathcal{P}]^{t}, \quad \text { with } t=l+\log \left|H^{1}\left(G, E(H)_{f}\right)\right|,
$$

where $\mathcal{P}$ is any prime factor of 2 in $K, l=\operatorname{rank}_{\mathbf{Z}}(E(F))$ is the $\mathbf{Z}$-rank of $E(F),\left|H^{1}\left(G, E(H)_{f}\right)\right|$ is the order of the first cohomology group $H^{1}\left(G, E(H)_{f}\right)$, and $G=\operatorname{Gal}(H / F)$.

\section{The Structure of the Mordell group $E(H)$.}

Lemma 1. The degree of the extension $H / F$ is $[H: F]=2$, where $F=$ $\mathbf{Q}(j(E)), H=K(j(E)), j(E)$ is the $j$-invariant of $E$.

Proof. Obviously we have $[H: F] \leq 2$. If $[H: F]=1$, then $K \subset F$. By a result in page 12-13 of [2] we know that $F=\mathbf{Q}(j(E))$ has a real embedding into the complex field $\mathbf{C}$. Since $K$ is totally imaginary, $K \subset F$ is impossible. Thus $[H: F]=2$. This proves the lemma.

Based on Lemma 1, we assume throughout the Galois group of $H / F$ to be $G=\operatorname{Gal}(H / F)=\{1, \sigma\}$. For any $\alpha \in \mathcal{O}_{K}$, let $[\alpha]$ denote the endomorphism of $E$ corresponding to the multiplication by $\alpha$. The multiplication by $\sqrt{-D}$ will be important to our following proof. Associating with $E: y^{2}=f(x)$, we consider the following elliptic curve

$$
E_{D}:-D y^{2}=f(x) \text {. }
$$

Note that $E_{D}$ and $E$ are isomorphic via the map

$$
i: E_{D}(\mathbf{C}) \rightarrow E(\mathbf{C}), \quad(x, y) \mapsto(x, \sqrt{-D} y) .
$$


Therefore we know that

$$
\operatorname{End}\left(E_{D}\right) \cong \operatorname{End}(E) .
$$

So $E_{D}$ also has complex multiplication by $\mathcal{O}_{K}$, and is defined over $F$. Also via the isomorphism $i$ of $E$ and $E_{D}$, we have

$$
E_{D}(F) \cong I
$$

where

$$
I=\{(x, \sqrt{-D} y) \mid(x, \sqrt{-D} y) \in E(H), x, y \in F\} .
$$

The subgroup $I$ of $E(H)$ defined here will be very important in the following analysis.

Lemma 2. The map $i \circ[\sqrt{-D}]$ is an F-isogeny of $E_{D}$ to E. Thus

$$
\operatorname{rank}_{\mathbf{Z}}\left(E_{D}(F)\right)=\operatorname{rank}_{\mathbf{Z}}(E(F))=l .
$$

Proof. By [1] we have

$$
[\sqrt{-D}](x, y)=(a(x), y \sqrt{-D} b(x)),
$$

with $a(x), b(x) \in F(x)$. So $i \circ[\sqrt{-D}]$ is an $F$-isogeny of $E_{D}$ to $E$.

Lemma 3. $\left(I_{f}:[\sqrt{-D}] E(F)_{f}\right)\left(E(F)_{f}:[\sqrt{-D}] I_{f}\right)=D^{l}$.

Proof.

$$
\begin{aligned}
D^{l} & =\left(E(F)_{f}:[D] E(F)_{f}\right) \\
& =\left(E(F)_{f}:[\sqrt{-D}] I_{f}\right)\left([\sqrt{-D}] I_{f}:[D] E(F)_{f}\right) \\
& =\left(E(F)_{f}:[\sqrt{-D}] I_{f}\right)\left(I_{f}:[\sqrt{-D}] E(F)_{f}\right) .
\end{aligned}
$$

Lemma 4. $2 E(H)_{f} \subset E(F)_{f} \oplus I_{f} \subset E(H)_{f}$, $\operatorname{rank}_{\mathbf{Z}}(E(H))=\operatorname{rank}_{\mathbf{Z}}(E(F))+\operatorname{rank}_{\mathbf{Z}}\left(E_{D}(F)\right)=2 \operatorname{rank}_{\mathbf{Z}}(E(F))=2 l$.

Proof. If $P=(x, y) \in E(F)_{f}$ with $P \in I_{f}$, then $y=0$, which means that $P$ is a torsion point. So $P=O$ is the point at infinity, and $E(F)_{f} \oplus I_{f}=E(F)_{f}+$ $I_{f} \subset E(H)_{f}$. For any $Q \in E(H)_{f}$, we have $2 Q=\left(Q+Q^{\sigma}\right)+\left(Q-Q^{\sigma}\right)$, where $G=\operatorname{Gal}(H / F)=\{1, \sigma\}$. Via the definition of $E(F)_{f}$ and $I_{f}$, we have

$$
E(F)_{f}=\left\{P \mid P^{\sigma}=P, \forall P \in E(H)_{f}\right\}, \quad I_{f}=\left\{P \mid P^{\sigma}=-P, \forall P \in E(H)_{f}\right\} .
$$

So $Q+Q^{\sigma} \in E(F)_{f}, Q-Q^{\sigma} \in I_{f}, 2 Q \in E(F)_{f} \oplus I_{f}$. Thus $2 E(H)_{f} \subset$ $E(F)_{f} \oplus I_{f} \subset E(H)_{f}$. This completes the proof.

As for the index of $E(F)_{f} \oplus I_{f}$ in $E(H)_{f}$, we have the following theorem, which could be also deduced from the cohomology theory of cyclic groups. 


\section{Theorem 1.}

$$
\left(E(H)_{f}: E(F)_{f} \oplus I_{f}\right)=\frac{2^{l}}{\left|H^{1}\left(G, E(H)_{f}\right)\right|},
$$

where $\left|H^{1}\left(G, E(H)_{f}\right)\right|$ is the order of the cohomology group $H^{1}\left(G, E(H)_{f}\right)$. Proof. Consider the colomology group

$$
H^{1}\left(G, E(H)_{f}\right)=Z^{1}\left(G, E(H)_{f}\right) / B^{1}\left(G, E(H)_{f}\right) .
$$

Let $T=\left\{P-P^{\sigma} \mid P \in E(H)_{f}\right\}$. We will prove that $Z^{1}\left(G, E(H)_{f}\right) \cong I_{f}$, $B^{1}\left(G, E(H)_{f}\right) \cong T$. For any cocycle $\xi \in Z^{1}\left(G, E(H)_{f}\right)$, let $\xi \stackrel{\phi}{\rightarrow} \xi_{\sigma}$, where $\operatorname{Gal}(H / F)=\{1, \sigma\}$. By the definition of cocycle we have that $0=$ $\xi_{1}=\xi_{\sigma^{2}}=\left(\xi_{\sigma}\right)^{\sigma}+\xi_{\sigma}$, so $\left(\xi_{\sigma}\right)^{\sigma}=-\xi_{\sigma}$, thus $\xi_{\sigma} \in I_{f}$, and $\phi$ is a map of $Z^{1}\left(G, E(H)_{f}\right)$ to $I_{f}$. Via the map $\phi$ we could see that $Z^{1}\left(G, E(H)_{f}\right) \cong$ $I_{f}, B^{1}\left(G, E(H)_{f}\right) \cong T$. Now consider the homomorphism $E(H)_{f} \stackrel{\psi=P-P^{\sigma}}{\longrightarrow}$ $T$. Obviously $2 I_{f} \subset T$. Since $\psi^{-1}\left(2 I_{f}\right)=E(F)_{f} \oplus I_{f}$, so

$$
\begin{aligned}
\left(E(H)_{f}: E(F)_{f} \oplus I_{f}\right)=\left(T: 2 I_{f}\right) & =\left(I_{f}: 2 I_{f}\right) /\left(I_{f}: T\right) \\
& =2^{l} /\left|H^{1}\left(G, E(H)_{f}\right)\right| .
\end{aligned}
$$

\section{Main Results and Their Proofs.}

We will first give a general theorem on a finitely-generated module over a Dedekind domain, which establishes a relationship between the Steinitz class and the index of the module in its corresponding free module. This theorem is the key to our final results about Steinitz class.

Theorem 2. Suppose that $L$ is a free $\mathcal{O}_{K}$-module, and $M \subset L$ is a submodule with $(L: M)<+\infty$. Then there is an integral $\mathcal{O}_{K}$-ideal $\mathcal{A}$ such that $[\mathcal{A}]$ is the Steinitz class of $M$, and $N_{\mathbf{Q}}^{K}(\mathcal{A})=(L: M)$, where $N_{\mathbf{Q}}^{K}(\cdot)$ denotes the norm map of ideals from $K$ to the rationals $\mathbf{Q}$.

Proof. Let $L=\bigoplus_{i=1}^{n} \mathcal{O}_{K} e_{i}$, so $\left\{e_{1}, \ldots, e_{n}\right\}$ is an $\mathcal{O}_{K}$-basis for $L$. We will inductively prove that there are $\mathcal{O}_{K}$-ideals $\mathcal{B}_{i}(i=1, \ldots, n)$ such that $M \cong \bigoplus_{i=1}^{n} \mathcal{B}_{i}$, and $(L: M)=\prod_{i=1}^{n}\left(\mathcal{O}_{K}: \mathcal{B}_{i}\right)$

When $n=1$, everything is obvious. Assume then the statement is true for $n-1$ and consider the module-homomorphism $\rho: L \rightarrow \mathcal{O}_{K}, \rho\left(\sum_{i=1}^{n} r_{i} e_{i}\right)=$ $r_{n}$. Then $\mathcal{B}=\rho(M)$ is an ideal of $\mathcal{O}_{K}$, and the sequence

$$
0 \rightarrow N \rightarrow M \stackrel{\rho}{\rightarrow} \mathcal{B} \rightarrow 0
$$


is exact, where $N=\operatorname{ker}(\rho) \cap M$. Since $\mathcal{B}$ is a projective $\mathcal{O}_{K}$-module, there exists $\mathcal{O}_{K}$-module $\mathcal{C} \subset M$ such that $\mathcal{C} \cong \mathcal{B}, \rho(\mathcal{C})=\mathcal{B}, M=N \oplus \mathcal{C} \cong N \oplus \mathcal{B}$. Thus

$$
(L: M)=(L: N \oplus \mathcal{C})=\left(L: \bigoplus_{i=1}^{n-1} \mathcal{O}_{K}+\mathcal{C}\right)\left(\bigoplus_{i=1}^{n-1} \mathcal{O}_{K}+\mathcal{C}: N \oplus \mathcal{C}\right)
$$

where $\left(L: \bigoplus_{i=1}^{n-1} \mathcal{O}_{K}+\mathcal{C}\right)=\left(\rho^{-1}\left(\mathcal{O}_{K}\right): \rho^{-1}(\mathcal{B})\right)=\left(\mathcal{O}_{K}: \mathcal{B}\right)$.

$$
{ }^{n-1} O_{K}=
$$

Consider $\mathcal{C} \cap \bigoplus_{i=1}^{n-1} \mathcal{O}_{K}=\mathcal{C} \cap \operatorname{ker}(\rho)$. When restricted on $\mathcal{C}$, the map $\rho$ is injective, so we have

$$
\begin{aligned}
\bigoplus_{i=1}^{n-1} \mathcal{O}_{K}+\mathcal{C} & =\bigoplus_{i=1}^{n-1} \mathcal{O}_{K} \oplus \mathcal{C} \\
\left(\bigoplus_{i=1}^{n-1} \mathcal{O}_{K}+\mathcal{C}: N \oplus \mathcal{C}\right) & =\left(\bigoplus_{i=1}^{n-1} \mathcal{O}_{K} \oplus \mathcal{C}: N \oplus \mathcal{C}\right) \\
& =\left(\bigoplus_{i=1}^{n-1} \mathcal{O}_{K}: N\right)
\end{aligned}
$$

Note that $N \subset \bigoplus_{i=1}^{n-1} \mathcal{O}_{K}$. So via the hypothesis of our induction, we know that there are $\mathcal{O}_{K}$-ideals $\mathcal{B}_{i}(i=1, \ldots, n-1)$ such that $N \cong \bigoplus_{i=1}^{n-1} \mathcal{B}_{i}$, and $\left(\bigoplus_{i=1}^{n-1} \mathcal{O}_{K}: N\right)=\prod_{i=1}^{n-1}\left(\mathcal{O}_{K}: \mathcal{B}_{i}\right)$. Thus we have $M \cong \bigoplus_{i=1}^{n} \mathcal{B}_{i}$ and $(L: M)=$ $\prod_{i=1}^{n}\left(\mathcal{O}_{K}: \mathcal{B}_{i}\right)=\prod_{i=1}^{n} N_{\mathbf{Q}}^{K}\left(\mathcal{B}_{i}\right)=N_{\mathbf{Q}}^{K}\left(\prod_{i=1}^{n} \mathcal{B}_{i}\right)$, where $\mathcal{B}_{n}=\mathcal{B}$. Now the proof is completed by the following lemma.

Lemma 5. Assume $\mathcal{A}_{1}$ and $\mathcal{A}_{2}$ are two nonzero ideals of the Dedekind domain $R$, then we have isomorphism of $R$-modules: $\mathcal{A}_{1} \oplus \mathcal{A}_{2} \cong R \oplus \mathcal{A}_{1} \mathcal{A}_{2}$.

Proof. See Lemma 13 in page 168 of [3].

We now intend to prove our main results via our Theorem 2. To use Theorem 2, we need first to find the corresponding $L$ and $M$ in the Mordell group $E(H)$. The corresponding $L$ is given in Lemma 6 . While the corresponding $M$ is given in the proofs of Theorem 4 and 5, i.e., $M=[\sqrt{-D}] E(H)_{f}$ if $D \equiv 3(\bmod 4) ; M=[2 \sqrt{-D}] E(H)_{f}$ if $D \equiv 1(\bmod 4)$.

Lemma 6. $L=\mathcal{O}_{K} \cdot E(F)_{f}$ is a free $\mathcal{O}_{K}$-module of rank $l$. 
Proof. Assume $P_{1}, \ldots, P_{l}$ form a $\mathbf{Z}$-basis of $E(F)_{f}$. We will prove

$$
L=\mathcal{O}_{K} \cdot E(F)_{f}=\bigoplus_{i=1}^{l} \mathcal{O}_{K} P_{i} .
$$

Now we suppose that $\sum_{i=1}^{l}\left[\alpha_{i}\right] P_{i}=0$ for some $\alpha_{i} \in \mathcal{O}_{K}(i=1, \ldots, l)$. When $D \equiv 3(\bmod 4)$, we have $\alpha_{i}=s_{i}+t_{i}(1+\sqrt{-D}) / 2\left(s_{i}, t_{i} \in \mathbf{Z}, i=1, \ldots, l\right)$, then via $\sum_{i=1}^{l}\left[\alpha_{i}\right] P_{i}=0$ we have $\sum_{i=1}^{l}\left[2 s_{i}+t_{i}\right] P_{i}=0$ and $\sum_{i=1}^{l}\left[\sqrt{-D} t_{i}\right] P_{i}=0$. Thus $t_{i}=0, s_{i}=0, \alpha_{i}=0(i=1, \ldots, l)$. This proves the theorem when $D \equiv 3(\bmod 4)$. The case $D \equiv 1(\bmod 4)$ goes in the same way.

To determine our corresponding $M$ in the case $D \equiv 3(\bmod 4)$, we need the following theorem.

Theorem 3. For $D \equiv 3(\bmod 4)$, we have $\left|H^{1}\left(G, E(H)_{f}\right)\right|=1$, and $E(H)_{f}$ $=\mathcal{O}_{K} \cdot E(F)_{f}+I_{f}$.

Proof. Let $P_{1}, \ldots, P_{l}$ form a $\mathbf{Z}$-basis of $E(F)_{f}$, and $Q_{1}, \ldots, Q_{l}$ form a Z-basis of $I_{f}$. Put $\alpha=(1+\sqrt{-D}) / 2$. We need only to prove that $E(H)_{f} /\left(E(F)_{f} \oplus I_{f}\right)=C_{1} \oplus \cdots \oplus C_{l}$, where $C_{i}=\left(\overline{[\alpha] P_{i}}\right)$ is subgroup of order 2 generated by $\overline{[\alpha] P_{i}}$ in the quotient group $E(H)_{f} /\left(E(F)_{f} \oplus I_{f}\right)$. (Here $\bar{a}$ denotes the residue class of $a$ in this quotient group.) Obviously we have $\overline{[\alpha] P_{i}} \neq \overline{0}$; otherwise there would be $t_{j}, s_{j} \in \mathbf{Z}(j=1, \ldots, l)$ such that $[\alpha] P_{i}=\sum_{j=1}^{l}\left[t_{j}\right] P_{j}+\sum_{j=1}^{l}\left[s_{j}\right] Q_{j}$, then $[1+\sqrt{-D}] P_{i}=\sum_{j=1}^{l}\left[2 t_{j}\right] P_{j}+\sum_{j=1}^{l}\left[2 s_{j}\right] Q_{j}$, and $P_{i}=\sum_{j=1}^{l}\left[2 t_{j}\right] P_{j}$, giving a contradiction.

Furthermore, if $\sum_{i=1}^{l}\left[u_{i}\right] \overline{[\alpha] P_{i}}=\overline{0}$ for some $u_{i} \in \mathbf{Z}(i=1, \ldots, l)$, then there are $t_{i}, s_{i} \in \mathbf{Z}(i=1, \ldots, l)$ such that $\sum_{i=1}^{l}\left[u_{i} \alpha\right] P_{i}=\sum_{i=1}^{l}\left[t_{i}\right] P_{i}+\sum_{i=1}^{l}\left[s_{i}\right] Q_{i}$, so

$$
\sum_{i=1}^{l}\left[u_{i}\right] P_{i}+\sum_{i=1}^{l}\left[u_{i} \sqrt{-D}\right] P_{i}=\sum_{i=1}^{l}\left[2 t_{i}\right] P_{i}+\sum_{i=1}^{l}\left[2 s_{i}\right] Q_{i} .
$$

Thus $\sum_{i=1}^{l}\left[u_{i}\right] P_{i}=\sum_{i=1}^{l}\left[2 t_{i}\right] P_{i}$, which gives $u_{i}=2 t_{i}(i=1, \ldots, l)$. Hence $\left[u_{i}\right] \overline{[\alpha] P_{i}}=\overline{\left[t_{i}\right][2 \alpha] P_{i}}=\overline{\left[t_{i}(1+\sqrt{-D})\right] P_{i}}=\overline{0}$. This completes the proof.

Now we can prove our main results via Theorem 2 . 
Theorem 4. Suppose that $D=p \equiv 3(\bmod 4)$ is a prime number, and $E$ is an elliptic curve having complex multiplication by the full ring $\mathcal{O}_{K}$ of integers of $K=\mathbf{Q}(\sqrt{-D})$. Then the Steinitz class of $E$ is the principal class, i.e., $\operatorname{St}(E)=1$.

Proof. Let $L=\mathcal{O}_{K} \cdot E(F)_{f}, M=[\sqrt{-p}] E(H)_{f}$. Since $M \cong E(H)_{f}$, we need only to prove $\operatorname{St}(M)$ is the principal class.

By Theorem 3 we have $E(H)_{f}=\mathcal{O}_{K} \cdot E(F)_{f}+I_{f}$. Thus

$$
\begin{aligned}
M=[\sqrt{-p}] E(H)_{f}=E(F)_{f} \cdot\left(\sqrt{-p} \mathcal{O}_{K}\right)+[\sqrt{-p}] I_{f} \subset \mathcal{O}_{K} \cdot E(F)_{f}=L & \\
(L: M) & =\left(\mathcal{O}_{K} \cdot E(F)_{f}:[\sqrt{-p}] E(H)_{f}\right) \\
& =\frac{\left(E(H)_{f}:[\sqrt{-p}] E(H)_{f}\right)}{\left(E(H)_{f}: \mathcal{O}_{K} \cdot E(F)_{f}\right)} \\
& =\frac{p^{l}}{\left(E(H)_{f}: \mathcal{O}_{K} \cdot E(F)_{f}\right)}
\end{aligned}
$$

Since $p$ is a prime number, so $(L: M)=p^{t}$ for some $t(0 \leq t \leq l)$. By Theorem 2, the Steinitz class of $M$ is equal to $[\mathcal{A}]$ for some $\mathcal{O}_{K}$-ideal $\mathcal{A}$, and $p^{t}=(L: M)=N_{\mathbf{Q}}^{K}(\mathcal{A})$. Since $p$ is a prime number, $\mathcal{A}=\left(\sqrt{-p} \mathcal{O}_{K}\right)^{t}$ is principal. Thus $\operatorname{St}(E)=\operatorname{St}(M)$ is the principal class.

Theorem 5. Suppose that $D=p \equiv 1(\bmod 4)$ is a prime number, and $E$ is an elliptic curve having complex multiplication by the ring $\mathcal{O}_{K}$ of all integers of $K=\mathbf{Q}(\sqrt{-D})$. Then the Steinitz class of $E$ is $\operatorname{St}(E)=[\mathcal{P}]^{t}$, where $[\mathcal{P}]$ is the ideal class of $K$ represented by $\mathcal{P}$ the prime factor of 2 in $\mathcal{O}_{K}, 2^{t}=2^{l}\left|H^{1}\left(G, E(H)_{f}\right)\right|$. In particular, the parity of $t$ determines $\operatorname{St}(E)$, since $\mathcal{P}$ is not principal while $\mathcal{P}^{2}=2 \mathcal{O}_{K}$ is principal.

Proof. Let $L=\mathcal{O}_{K} \cdot E(F)_{f}, M=[2 \sqrt{-p}] E(H)_{f}$. Since $M \cong E(H)_{f}$, so $\operatorname{St}(E)=\operatorname{St}(M)$. Note that $[2 \sqrt{-p}] E(H)_{f} \subset[\sqrt{-p}]\left(E(F)_{f} \oplus I_{f}\right),[\sqrt{-p}] I_{f} \subset$ $E(F)_{f}$. Thus we have $M \subset \mathcal{O}_{K} \cdot E(F)_{f}=L$, and

$$
\begin{aligned}
(L: M) & =\left(\mathcal{O}_{K} \cdot E(F)_{f}:[2 \sqrt{-p}] E(H)_{f}\right) \\
& =\frac{\left(E(H)_{f}:[2 \sqrt{-p}] E(H)_{f}\right)}{\left(E(H)_{f}: \mathcal{O}_{K} \cdot E(F)_{f}\right)} \\
& =\frac{(4 p)^{l}}{\left(E(H)_{f}: E(F)_{f} \oplus I_{f}\right)\left(E(F)_{f} \oplus I_{f}: \mathcal{O}_{K} \cdot E(F)_{f}\right)} \\
& =\frac{(4 p)^{l}}{2^{l}\left|H^{1}\left(G, E(H)_{f}\right)\right|^{-1}\left(I_{f}:[\sqrt{-p}] E(F)_{f}\right)} \\
& =2^{l}\left|H^{1}\left(G, E(H)_{f}\right)\right| \cdot p^{l} /\left(I_{f}:[\sqrt{-p}] E(F)_{f}\right) .
\end{aligned}
$$

Thus $(L: M)=2^{t} p^{r}$ for some $t, r \geq 0$, since $p$ is a prime number. By Theorem 2 we know that $N_{\mathbf{Q}}^{K}(\mathcal{A})=2^{t} p^{r}$ for some $\mathcal{O}_{K}$-ideal $\mathcal{A}$. Therefore $\mathcal{A}=\mathcal{P}^{t}\left([\sqrt{-p}] \mathcal{O}_{K}\right)^{r}, \operatorname{St}(E)=[\mathcal{A}]=\left[\mathcal{P}^{t}\right]$. This proves the theorem. 
Corollary 1. Suppose as in Theorem 5. If $l=\operatorname{rank}_{\mathbf{Z}}(E(F))=1$, then $H^{1}\left(G, E(H)_{f}\right)$ determines the Steinitz class of $E$.

Now we analyze the examples of Dummit and Miller in [1] by utilizing the above method. For these examples, we have $K=\mathbf{Q}(\sqrt{-10}), D=10$, $H=K(\sqrt{5})=\mathbf{Q}(\sqrt{-10}, \sqrt{5})$. We consider the $\mathcal{O}_{K}$-module $L=\mathcal{O}_{K} \cdot E(F)_{f}$ and $M=2[\sqrt{-10}] E(H)_{f}$. Then via the same idea in the proof of Theorem 5 we have similar ratiocination for $D=10$ :

$$
\begin{aligned}
(L: M) & =\frac{\left(E(H)_{f}: 2[\sqrt{-10}] E(H)_{f}\right)}{\left(E(H)_{f}: \mathcal{O}_{K} \cdot E(F)_{f}\right)} \\
& =\frac{(4 \cdot 10)^{l}}{\left(E(H)_{f}: E(F)_{f} \oplus I_{f}\right)\left(E(F)_{f} \oplus I_{f}: \mathcal{O}_{K} \cdot E(F)_{f}\right)} \\
& =\frac{(40)^{l}}{2^{l}\left|H^{1}\left(G, E(H)_{f}\right)\right|^{-1}\left(I_{f}:[\sqrt{-10}] E(F)_{f}\right)} \\
& =2^{l}\left|H^{1}\left(G, E(H)_{f}\right)\right| 10^{l} /\left(I_{f}:[\sqrt{-10}] E(F)_{f}\right) .
\end{aligned}
$$

Thus the Steinitz class of $E$ is determined by the 2-exponent of

$$
2^{l}\left|H^{1}\left(G, E(H)_{f}\right)\right|\left(I_{f}:[\sqrt{-10}] E(F)_{f}\right) .
$$

(DM1) Consider the following elliptic curve of Dummit and Miller in [1]:

$$
E_{1}: y^{2}=x^{3}+(6+6 \sqrt{5}) x^{2}+(7-3 \sqrt{5}) .
$$

Then $l=1,\left|H^{1}\left(G, E(H)_{f}\right)\right|=2,\left(I_{f}:[\sqrt{-10}] E(F)_{f}\right)=1$. Therefore we know that $2^{l}\left|H^{1}\left(G, E(H)_{f}\right)\right|\left(I_{f}:[\sqrt{-10}] E(F)_{f}\right)=4$. Thus the Steinitz class of $E_{1}$ is the principal class, i.e., $\operatorname{St}\left(E_{1}\right)=1$.

(DM2) Consider the following elliptic curve in [1]:

$$
E_{1, \text { isog }}: y^{2}=x^{3}-(912+12 \sqrt{5}) x^{2}+(188+84 \sqrt{5}) x .
$$

We have $l=1, \quad\left|H^{1}\left(G, E(H)_{f}\right)\right|=2, \quad\left(I_{f}:[\sqrt{-10}] E(F)_{f}\right)=2$, and $2^{l}\left|H^{1}\left(G, E(H)_{f}\right)\right|\left(I_{f}:[\sqrt{-10}] E(F)_{f}\right)=2^{3}$. Thus the Steinitz class $\operatorname{St}\left(E_{1, \text { isog }}\right)=[\mathcal{P}]$, where $\mathcal{P}$ is a prime factor of 2 in $\mathcal{O}_{K}$.

(DM3) For $E_{3}: y^{2}=x^{3}+36 x^{2}+(162-72 \sqrt{5}) x$, in [1] , we have $l=$ 2, $\left|H^{1}\left(G, E(H)_{f}\right)\right|=2, \quad\left(I_{f}:[\sqrt{-10}] E(F)_{f}\right)=1,2^{l}\left|H^{1}\left(G, E(H)_{f}\right)\right|\left(I_{f}:\right.$ $\left.[\sqrt{-10}] E(F)_{f}\right)=2^{3}$. Thus $\operatorname{St}\left(E_{3}\right)=[\mathcal{P}], \mathcal{P}$ a prime factor of 2 in $\mathcal{O}_{K}$.

There are still many open problems about the Steinitz classes of elliptic curves. For example, we have the following conjecture.

Conjecture. Both the cases $\operatorname{St}(E)=1$ and $\operatorname{St}(E) \neq 1$ exist for some elliptic curves $E$ having complex multiplication by $\mathcal{O}_{K}$, where $K=\mathbf{Q}(\sqrt{-D})$ with prime number $D \equiv 1(\bmod 4)$. 


\section{References}

[1] D.S. Dummit and W.L. Miller, The Steinitz class of the Mordell-Weil group of some CM elliptic curves, J. Number Theory, 56 (1996), 52-78.

[2] B. Gross, Arithmetic on Elliptic Curves with Complex Multiplication, SLN, 776, Springer-Verlag, Berlin, 1980.

[3] F. Keqin, Introduction to Commutative Algebra, Higher Education Press, Beijing, 1985.

[4] G. Shimura, Introduction to the Arithmetic Theory of Automorphic Functions, Princeton Univ. Press, 1971.

[5] J.H. Silverman, The Arithmetic of Elliptic Curves, Spring-Verlag, New York, 1982.

Received September 3, 1998 and revised February 10, 1999.

Tsinghua University

BEIJING 100084

P.R. CHINA

TSINGHUA UNIVERSITY

BEIJING 100084

P.R. China

E-mail address: xianke@tsinghua.edu.cn 